\section{Mit topischen Steroiden den Heuschnupfen bekämpfen}

\section{Leiden Heuschnupfenpatienten massiv unter ihrer Allergie, empfiehlt die International Rhinitis Management Working Group topische Steroide. Bei ganzjähriger allergischer Erkrankung der oberen Atemwege sind sie Mittel der Wahl.}

Bei Kindern rangieren die intranasal applizierten Kortikoide gleich nach topischem Dinatriumcromoglicat DNCG oder nicht-sedierenden Antihistaminika. Auf dieses Behandlungsschema hat R. Mösges, Köln, hingewiesen.

Bei saisonalen, mäßig starken Beschwerden ist nach Angaben des Allergologen die Behandlung mit Mastzellstabilisatoren oder Degranulationshemmern wie DNCG oder Nedocromil ausreichend. Sie kann bei kurzfristigen Belastungen durch topische Antihistaminika ergänzt werden. Bei chronischer Entzündung der Schleimhaut sind Steroide unerläßlich.

\section{Nasale Obstruktion: Vorteile für Kortikoide}

Haben erwachsene Patienten starke Beschwerden mit Niesen, Juckreiz, hen nach Ansicht von G. Kunkel, Berlin, topische Steroide ganz im Vo r$\mathrm{d}$ e rgrund. „Im Ve rgleich zu oralen Antihistaminika wirken die topischen Steroide besser bei Schnupfen und Obstruktion, das Niesen wird durch beide Substanzgruppen ähnlich gut unterdrückt", so Kunkel. In der Initialbehandlung können zusätzlich Vasokonstriktoren verabreicht werden. Als einzige kausale Therapieform steht schließlich die spezifische Immuntherapie zur Verfügung.

Topische Steroide sind nach Einschätzung beider Referenten häufig Mittel der Wahl bei Patienten mit allergischer und perennialer Rhinitis - dennoch gibt es Vorbehalte, weil die Substanzen nur langsam wirken und ihre schleimhaut austrocknen und so $\mathrm{zu}$ Krustenbildung und Nasenbluten führen kann. Die Steroidtherapie kann Fließschnupfen und Obstruktion, steintranasale Anwendung die Nasen- aber nach Angaben Mösges durchaus w e i t e rgeführt werden, wenn man

- die Dosis reduziert,

- pflegende Nasensalben verwendet,

- von einem Aerosolpräparat auf ein Spray umsteigt und

- nur einmal täglich sprüht.

Neues nasales Steroid mit innovativer Galenik

Den Worten Mösges zufolge ist für die Patientencompliance von entscheidender Bedeutung, wie ein Präparat riecht oder schmeckt. Aus diesen Gründen lehnen einige Patienten die Applikation topischer Steroide ab. Ein neues wäßriges Nasenspray $\left(\right.$ Nasacort $\left.^{\circledR}\right)$ mit dem bewährten Steroid Tr i a m c i n o l o nacetonid (TAA) zeichnet sich durch eine Geruchs- und Geschmacksneutralität aus. Die thixotropen Eigenschaf- ten der Zubereitung bewirken eine längere Verweildauer auf der Nasenschleimhaut, worauf G. Rasp, München, hinwies. Nach Applikation der Lösung in die Nase erlangt diese wieder ihre ursprünglich höhere Viskosität und läuft nicht in den Rachen hinab.

\section{Triamcinolonacetonid: Besserung} der Symptome ab dem ersten Tag In einer Dosierung von $220 \mu \mathrm{g}$ TA A einmal täglich bessern sich die $\mathrm{Heu}-$ schnupfensymptome bereits ab dem ersten Tag im Ve rgleich zu Plazebo deutlich. TAA beeinträchtigt in therapeutischer Dosierung nicht die Nebennierenrindenfunktion und eignet sich zur Behandlung von Erwachsenen und Kindern mit saisonaler oder jahreszeitlich unabhängig auftretender allerg ischer Rhinitis. Dabei kann die Dosis um die Hälfte reduziert werden, sobald die Beschwerden unter Kontrolle sind. Auch potenten Antihistaminika ist das topische Steroid ab der zweiten Behandlungswoche in der Wirksamkeit signifikant überlegen, erklärte Rasp abschließend.

(bg) Quellen: Satellitensymposium und Pressegespräch im Rahmen
des 25. Kongresses des Ärzteverbandes Deutscher Allerg o 1 ogen, Göttingen, September 1998, sowie Satellitensymposium im Rahmen der 32. Fortbildungsveranstaltung HNO, Hannov e r, Oktober 1998, jeweils unterstützt von der Firma RhônePoulenc Rorer.

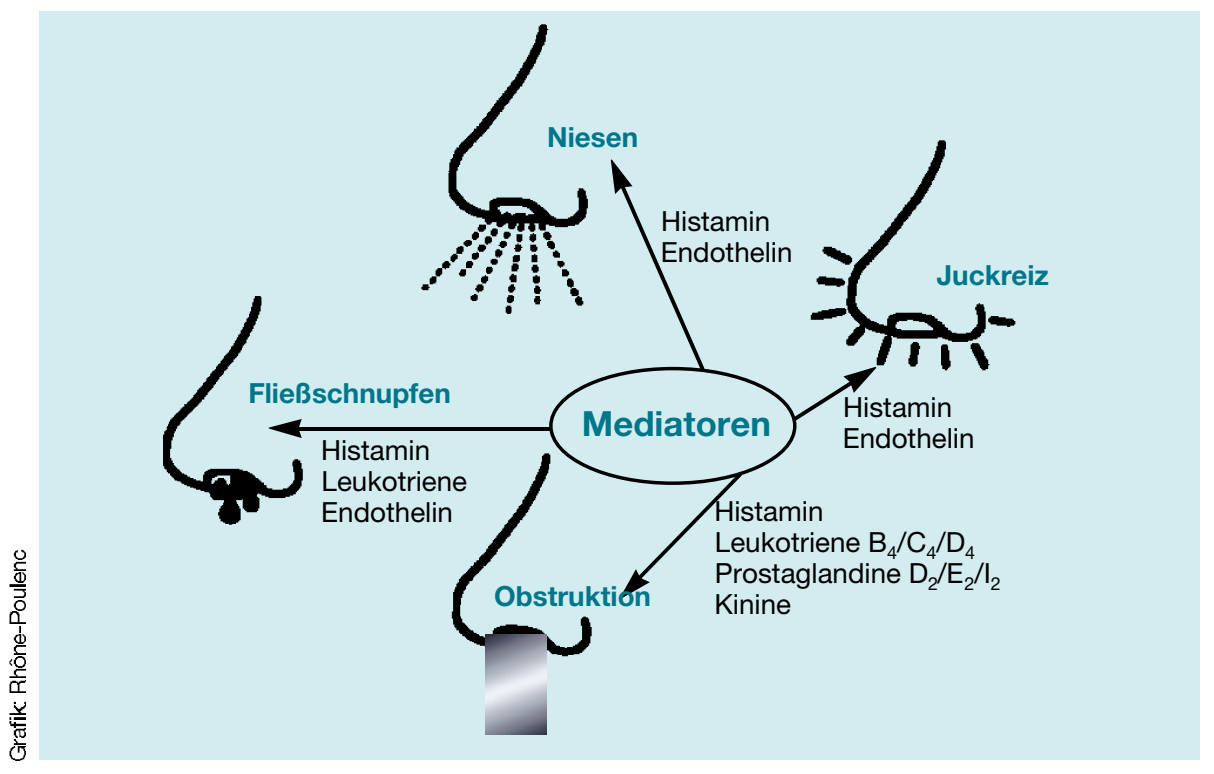

Pathomechanismus der allergischen Rhinitis auf der Ebene von Zellmediatoren: Aus Mastzellen, Eosinophilen und Basophilen werden verschiedene Entzündungsmediatoren freigesetzt, die dann die typischen Heuschnupfensymptome hervorrufen. 